\title{
ANÁLISE DA CLIENTELA QUE CONSULTOU EM 1973 NA FACULDADE DE ODONTOLOGIA \\ DA UNIVERSIDADE FEDERAL DO RIO GRANDE DO SUL E QUE DEIXOU DE RECONSULTAR EM 1974*
}

\author{
Suzana Gomes Moreira **
}

MOREIRA, Suzana Gomes. Análise da clientela que consultou em 1973 na Faculdade de Odontologia da Universidade Federal do Rio Grande do Sul e que deixou de reconsultar em 1974. Revista da Faculdede de Odontologia, Porto Alegre, 21-22-23-24: 53-65, 1979/82.

\section{Descritores: PACIENTES, ANÁLISE DA DEMANDA NA FACULDADE DE ODONTOLOGIA DA UFRGS, 1973. ODONTOLOGIA SOCIAL}

\section{RESUMO}

Foram entrevistados trezentos e quinze pacientes que consultaram na Faculdade de Odontologia da UFRGS no ano base de 1973 e foram identificadas as principais causas do nếo retorno em 1974. Analizaram-se fatores relevantes como: caracterizaço da clientela em dados pessoais e socio-econômicos, fatores que influenciaram a utilização dos serviços ambulatoriais da Faculdade. opiniz̃o sobre o tratamento recebido e posicionamento quanto a futura reconsulta na Faculdade. "Vários cruzamentos stó feitos para estudo de associaçắo entre os fatores. As principais razóes apresentadas para năo reconsultar foram: falta de tempo do paciente, ausência de necessidades de tratamento odontológico e procura de outro dentista.

\section{INTRODUÇÃO E REVISÃO DA LITERATURA}

Em Odontologia vêm-se realizando trabalhos que analisam a utilizacấo dos serviços prestados nas Faculdades ou Escolas de Odontologia. Entre os aspectos considerados ou examinados encontram-se os fatores que atraem as pessoas a buscar os seus serviços 3,7,8,10 o perfil da população atendida nas escolas; $1,2,5,7,10$ os aspectos considerados pelos pacientes como agradáveis ou desagradáveis na escola ou no tratamento; 3,6 a pretensão a continuar freqüentando as clínicas 3 caso o custo do tratamento fosse igual ao das clínicas par-

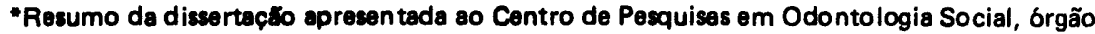
auxiliar da Faculdade de Odontologia da UFRGS, com vistas a obtenção do título de Mestre em Odontologia.

- Mestre en Odontologia e Professora da Faculdade de Odontologia da UFRGS.
} 
ticulares, 6,8 razões determinantes da interrupcão do tratamento ou da sua iniciação. 4,8

As causas que deram origem às pesquisas sobre utilização de serviços das Frnildades de Odontologia prendem-se de modo geral, a necessidade de, através de uma melhor compreensão deste fenômeno, adotar providências no sentido de obter um fluxo cont/nuo e suficiente de pacientes para o ensino.

A criação do Serviço de Triagem da Faculdade de Odontologia da UFRGS em 1971, centralizou o sistema de ingresso de pacientes para o ensino. Os primeiros resultados obtidos com o sistema de computação de dados então implantado, permitiu que, entre outros aspectos, se identificassem que o grau de reconsultas de pacientes era extremamente baixo. Assim, durante os anos letivos de 1972 e 1973, matricularam-se no Serviço de Triagem 7125 pessoas e, em 1974 retornaram apenas 590 , equivalendo a menos a $10 \%$ da população que demandou a Faculdade nos dois referidos anos.

Analisando parcialmente estas informações, verificou-se que em um ano (1973), 2669 pessoas procuraram a Faculdade e destas, 277 reconsultaram em 1974.

Este fato, aliado à crescente necessidade de pacientes para o ensino, decorrente da expansão das atividades clínicas dentro do currículo e do aumento do número de alunos, levou a administração da Faculdade a preocupar-se com o problema.

E evidente que medidas visando a solucionato deveriam estar intima. mente associadas ao conhecimento exato das razőes que levavam os pacientes à adoção deste tipo de con. duta.
Para encontrar explicaçóes mais precisas para o problema levantado, desenvolveu-se uma pesquisa domiciliar por amostragem com os pacientes que se registraram em 1973 e não retornaram em 1974. Foi realizado um estudo desta clientela, no qual se levantou $e$ analisou vários fatores que permitiram, com a cautela necessária, se inferir sobre as ocorrências gerais. O presente trabalho apresenta os principais tópicos levantados e analisados neste estudo.

\section{OBJETIVOS}

\section{1 - Objetivo Geral}

Através de pesquisa domiciliar, por amostragem e entrevista pessoal, identificar as principais causas do não retorno, para reconsulta, aos serviços ambulatoriais da Faculdade de Odontologia da UFRGS, dos pacientes que nele se inscreveram em 1973 e não reconsultaram até fim de 1974.

\section{2 - Objetivos especificos}

Caracterizar a clientela, objeto da pesquisa, quanto a:

\subsection{1 - Composição segundo:}

- idade, sexo, estado civil;

- ocupaçáo, grau de instrucão, rendimento individual, direito a assistência odontologica.

2.2.2 - Condicionantes da utilização dos serviços da Faculdade, traduzidos pela opiniăo sobre:

- finalidade da prestação de serviços pela Faculdade de Odontologia;

- competência do estudante de Odontologia para prestar atendimentos; 
- preços cobrados pela Faculdede;

- acessibilidade física à Faculdade; .

- razóos determinantes da preferência por atendimentos na Faculdade em 1973.

2.2.3 - Opiniśo da clientela sobre o tratamento recebido na Fa. culdade, em 1973, quanto a:

- recebimento ou nio de atendimentos na Faculdade em 1973;

- resolucão ou não do problema que determinou a procura da Faculdade;

- fato de ter ou nåo completado o tratamento;

- grau de satisfatoriedade com relação ao tratamento recebido;

- discrepância entre a expectativa a forma de atendimento, na Faculdade, em 1973.

2.2.4 - Razóos apresentadas para nåo reconsultar na Faculdade em 1974, desdobradas em:

- proporcão dos que não retornaram;

- razס̄es apresentadas para o não retorno;

- recebimento ou não de orientaç̃o para conti-, nuar o tratamento;

- busca de atendimento por outro dentista após consulta, na Faculdade, em 1973;

- influência da localização da Faculdade na não reconsulta.

\subsection{5 - Posicionamento quanto a futura reconsulta na Facul- dade.}

\section{MATERIAL E MÉTODOS}

População estudada: Como indicador, com as reservas que esta circunstância poderia implicar, tomou-se, para estudo, o conjunto de pacientes que procuraram os servicos ambulatorias da Faculdade em 1973, se registraram no Serviço de Triagem da referida instituição naquele ano $e$, tendo ou não recebido tratamento, não retornaram em 1974 (correspondendo a 2.392 pessoas segundo arquivo da Faculdade).

Deste conjunto de pacientes foi selecionada uma amostra aleatória simples para pesquisa de atributos, para um nível de significância de 5\% 15 pontos de percentagem para 0 fator comando), e considerando-se como comando para o dimensionamento as proporções das causas que justificassem a maior parte do não retorno em 1974.

Estudaram-se 315 pacientes entre 2.392, representando aproximadamente uma amostra de $13,17 \%$ dos pacientes registrados em 1973 e que nåo retornaram em 1974.

Procedimentos referentes ao levantamento dos dados: A coleta dos dados foi realizada através de entrevistas pessoais com os constituintes de amostra no local fornecido como de residência na época de registro no Serviço de Triagem, mediante o preenchimento de um formulário constituído de 85 questơes do tipo aberta e fechada.

As entrevistas, do tipo estruturada, foram efetuadas por uma equipe de estudantes universitários, predo. minantemente do curso de Odontolo- 
gia. Duravam em média uma hora. Foram feitas revisitas, planejadas, até a concretização da entrevista.

O trabalho de campo foi desenvolvido, em duas etapas, de agosto a dezembro de 1975.

Na primeira etapa a amostra foi estimada arbitrariamiente em. 70 pacientes dos que eram objeto, de estudo. Esta amostra piloto permitiu dimensionar o tamanho total da amostra em 809 pacientes.

Como 70 fizeram parte da primeira fase, a amostra da segunda etapa ficou constituída por 739 pessoas. Nas duas fases observou-se a mesma metodologia e utilizou-se o mesmo instrumento de pesquisa.

Dos 809 pacientes selecionados, 88 กล̃o puderam ser procurados por insuficiência de dados sobre o endereço na ficha de registro. Ao final do trabalho de campo, constatou-se que vinte pessoas haviam retornado em 1974, porém sem terem passado pelo Serviço de Triagem, três se recusaram a conceder a entrevista e que 383 pacientes foram procurados e não localizados por várias razões, como por exemplo: mudança para local ignorado ou fora da Grande Porto Alegre, desconhecidos no endereço, em viagem prolongada, etc. Desta forma, a população a ser estudada ficou reduzida a 315 pessoas.

\section{RESULTADOS E DISCUSSÃO}

A organização dos resultados $\theta$ discussfó obedeceu à seguinte ordem:

1 - Caracterizaça da clientela;

2 - Condicionantes da utilização dos servicos ambulatoriais da Faculdade em 1973;
3 - Opinião da clientela sobre o tratamento recebido na Faculdade de Odontologia em 1973;

4 - Razões para não reconsultar na Faculdade de Odontologia em 1974;

5 - Posicionamento quanto a futura consulta na Faculdade de Odontologia.

\section{1 - Caracterização da clientela}

\section{Dados pessoais}

Na população estudada há predominância de clientes na faixa etária de 15 a 34 anos, pois perfazem dois terços dos que não retornaram. Sa. lienta-se que o grupo etário modal é entre 15 e 24 anos e a idade mediana 29 anos.

Dois terços são de sexo feminino contra um terço do sexo masculino. Aproximadamente metade são solteiros, enquanto pouco mais de um ter. Co são casados.

\section{Dedos scio-econômicos}

Para a classificaç̃o das ocupações utilizou-se escala ocupacional usada na pesquisa sobre "O Sistema Educacional e o Mercado de Trabalho na Região Metropolitana de Porto Alegre (Projeto 01), realizada em 1976.9

Nesta escala, as ocupaçס̃es estão distribuidas em dez níveis. A tabola 1 mostra a distribuição dos pacientes que não retornaram segundo a respectiva ocupação no momento da coleta de dados.

Como norma classificou-se a ocupaçăo que o paciente exercia na época da coleta de dados. Quando o paciente era estudante, nåo exercendo nenhuma atividade remunerada, considerou-se a ocupação do pai ou responsável, o que ocorreu com 44 pacientes. Similarmente, no caso de 53 pacientes que se dedicavam unica- 
mente a afazeres domésticos e eram casadas, utilizou-se, para efeito de classificação do status social, a ocupaçăo do marido.

A ocupação modal é manual qualificado (29\%), seguida por manual nåo qualificado (27\%). Predominância da ocupaçåo manual (56\%).

TABELA 1 - PACIENTES QUE NAO RETORNARAM, CONFORME NATUREZA DAS OCUPAÇOES. PORTO ALEGRE - 1975.

\begin{tabular}{|c|c|c|c|c|}
\hline NATUREZA DAS OCUPAÇOES & NÚMERO & $\underset{\%}{\text { PROPORCAXO }}$ & $\begin{array}{c}\text { PROPORCAO } \\
\text { ACUMULADA } \\
\%\end{array}$ & $\begin{array}{c}\text { PROPORCAAO } \\
\text { DESACUMULADA } \\
\%\end{array}$ \\
\hline 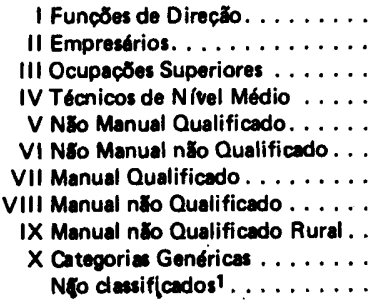 & $\begin{array}{r}8 \\
12 \\
9 \\
21 \\
43 \\
5 \\
91 \\
84 \\
3 \\
26 \\
13\end{array}$ & $\begin{array}{r}2,54 \\
3,81 \\
2,86 \\
6,67 \\
13,65 \\
1,59 \\
28,89 \\
26,66 \\
0,95 \\
8,25 \\
4,13\end{array}$ & $\begin{array}{r}2,54 \\
6,35 \\
9,21 \\
15,88 \\
29,53 \\
31,12 \\
60,01 \\
86,67 \\
87,62 \\
95,87 \\
100,00\end{array}$ & $\begin{array}{r}100,00 \\
97,46 \\
93,65 \\
90,79 \\
84,12 \\
70,47 \\
68,88 \\
39,99 \\
13,33 \\
12,38 \\
4,13\end{array}$ \\
\hline TOTAL. . . . . . . . . . . & 315 & 100,00 & & \\
\hline
\end{tabular}

1 Por insuficiôncia de informaçốes

A tabela 2 mostra a distribuição dos pacientes conforme grau de instrução. $O$ critério adotado implicitamente admite o enquadramento de graus paralelos, de cursos diferentes, como cursos técnicos, por exemplo.

TABELA 2 - PACIENTES QUE NAO RETORNARAM, CONFORME GRAU DE INSTRUÇO. PORTO ALEGRE. 1975.

\begin{tabular}{|c|c|c|c|c|}
\hline GRAU DE INSTRUÇĀO & NÚMERO & $\underset{\%}{\text { PROPORÇÃO }}$ & $\begin{array}{c}\text { PROPORCÃO } \\
\text { ACUMULADA } \\
\%\end{array}$ & $\begin{array}{c}\text { PROPORÇÅO } \\
\text { DESACUMULADA } \\
\%\end{array}$ \\
\hline 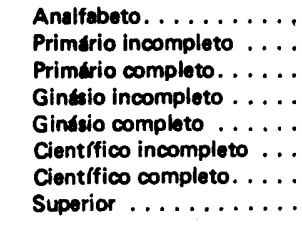 & $\begin{array}{l}7 \\
58 \\
79 \\
48 \\
48 \\
30 \\
27 \\
18\end{array}$ & $\begin{array}{r}2,22 \\
18,41 \\
25,09 \\
15,24 \\
15,24 \\
9,52 \\
8,57 \\
5,71\end{array}$ & $\begin{array}{r}2,22 \\
20,63 \\
45,72 \\
60,96 \\
76,20 \\
85,72 \\
94,29 \\
100,00\end{array}$ & $\begin{array}{r}100,00 \\
97,78 \\
79,37 \\
54,28 \\
39,04 \\
23,80 \\
14,28 \\
5,71\end{array}$ \\
\hline TOTAL . . . . . . . & 315 & 100,00 & & \\
\hline
\end{tabular}


Predominam os pacientes com grau de instrução primária completa ou acima (79\%). O grau de instrução modal é primária completo (um quarto dos pacientes). Quanto aos de curso superior $(6 \%)$, possivelmente são universitários que se valem do serviço.

A tabela 3 mostra a distribuição dos pacientes que não retornaram conforme renda individual, em valores de 1975. Destaca-se que ém um terço dos casos a renda individual modal é de 0,2 a menos de 1,4 salários mínimos

$(\mathrm{Cr} \$ 100,00 \vdash \mathrm{Cr} \$ 700,00)$;

Há predominância da renda abaixo de 1,4 salários mínimos $(\mathrm{Cr} \$ 700,00)$ em quase dois terços dos casos:

A renda individual mediana é 0,9 salários mínimos (pelo menos $50 \%$ dos individuos ganham menos de 1 salário mínimol. O salário mínimo em 1975 era $\mathrm{Cr} \$ 495,00$.

Quase um terço não tinha rendimento.

TABELA 3 - PACIENTES QUE NÃO RETORNARAM, CONFORME RENDA INDIVI. DUAL. PORTO ALEGRE - 1975 *

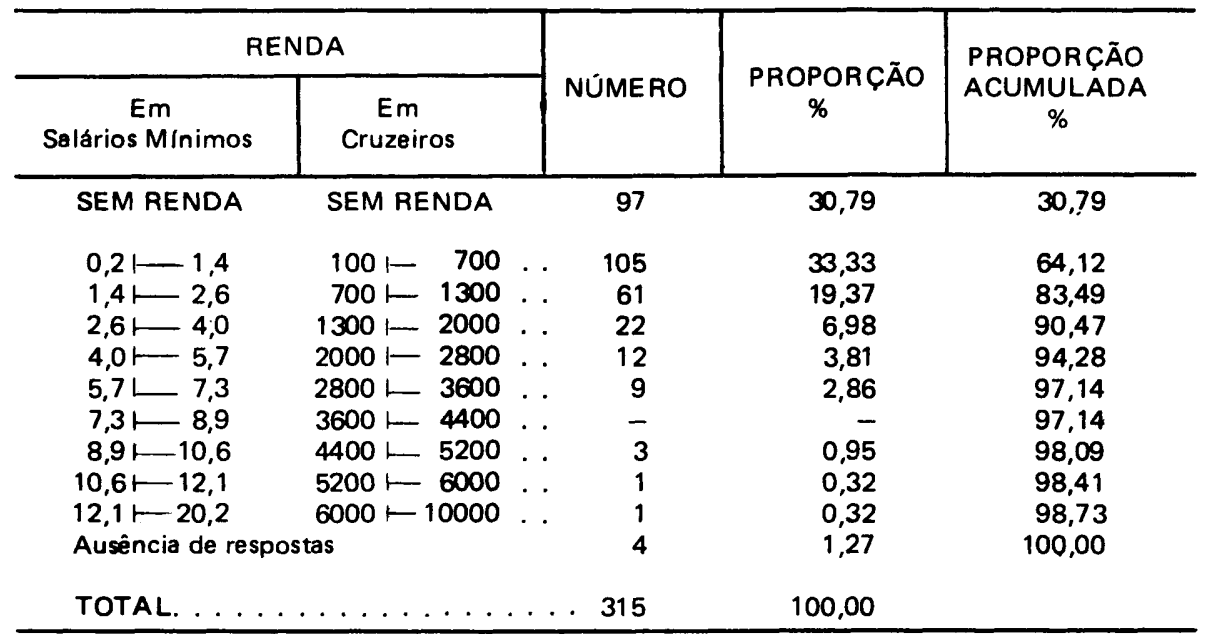

•Em valores de 1975, quando o salário mínimo era de $\operatorname{Cr} \$ 495,00$. 
A tabela 4 mostra a distribuição dos pacientes que não retornaram, de acordo com a circunstância de terem ou não direito a assistência odontológica em instituições, e o número de entidades assistenciais das quais se podem valer.

TABELA 4 - PACIENTES QUE NÃO RETORNARAM, CONFORME NÚMERO DE ENTIDADES ASSISTENCIAIS EM ODONTOLOGIA A QUE TÉM DIREITO. PORTO ALEGRE - 1975.

\begin{tabular}{|c|c|c|c|c|}
\hline $\begin{array}{l}\text { TEM DIREITO A ASSISTENCIA } \\
\text { ODONTOLOGICA EM: }\end{array}$ & NÚMERO & $\begin{array}{c}\text { PROPORÇÃO } \\
\%\end{array}$ & $\begin{array}{c}\text { PROPORÇAO } \\
\text { ACUMULADA } \\
\% \\
\end{array}$ & $\begin{array}{c}\text { PROPORÇÃo } \\
\text { DESACUMULADA } \\
\%\end{array}$ \\
\hline $\begin{array}{l}\text { Não tem Direito } \ldots \ldots \ldots \\
\text { Uma entidade . . . . . . . } \\
\text { Duas entidades } \ldots \ldots \ldots \\
\text { Três entidades. . . . . . . } \\
\text { Quatro entidades. . . . }\end{array}$ & $\begin{array}{r}54 \\
188 \\
50 \\
20 \\
3\end{array}$ & $\begin{array}{r}17,14 \\
59,69 \\
15,87 \\
6,35 \\
6,95\end{array}$ & $\begin{array}{r}17,14 \\
76,83 \\
92,70 \\
99,05 \\
100,00\end{array}$ & $\begin{array}{r}100,00 \\
82,86 \\
23,17 \\
7,30 \\
0,95\end{array}$ \\
\hline TOTAL . . . . . . . . & 315 & 100,00 & & \\
\hline
\end{tabular}

Observa-se que: o número modal de entidades é uma $(60 \%)$; embora quase um quinto dos pacientes não dispusesse de entidades assistenciais, a grande maioria dispõe de pelo menos uma entidade. A tabela 5 apresenta as principais entidades disponíveis, com assistência odontológica, aos pacientes que não retornaram.

TABELA 5 - ENTIDADES ASSISTENCIAIS ODONTOLOGICAS DISPONIVEIS AOS PACIENTES QUE NAOO RETORNARAM. PORTO ALEGRE - 1975.

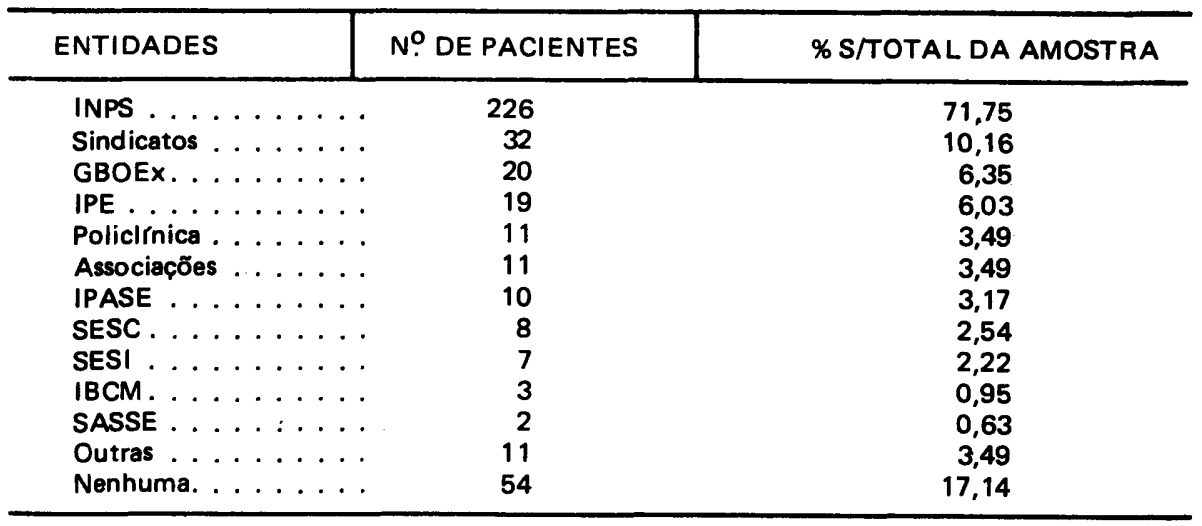


Com grande destaque apareceu o INPS como a entidade que dá maior cobertura aos pacientes entrevistados, atingindo $72 \%$ dos mesmos.

\section{2 - Condicionantes da utilização dos serviços ambulatoriais da Faculdade de Odontologia em 1973.}

Entre os pacientes que não retornaram, três quartos (74\%) informaram que a prestação de serviços odontológicos à comunidade pela Faculdade visa propiciar treinamento aos estudantes, embora alguns admitissem também benefício à população que não tem condições financeiras para pagar um dentista particular. $(25 \%)$.

Com relação a possibilidade de um estudante de Odontologia prestar atendimentos, comparando-o a um profissional formado, dois terços dos que não retornaram admitem que o estudante pode prestar atendimento igual ao dentista formado, sem apresentar qualquer restrição.

Considerando os preços cobrados pela Faculdade para a prestação de serviços odontológicos, três quartos dos pacientes opinaram que os acham baratos $(77 \%)$. Juntando-se com os que acham os preços razoáveis, somam $93 \%$ dos pacientes entrevistados.

Quanto a dificuldade de acesso $f$ ísico a Faculdade, considerando o lugar em que mora, a grande maioria (91\%) informou que não há dificuldade.

Todos os pacientes objeto de estudo consultaram em 1973. Admitindo-se isto como preferência a não ter consultado em outro lugar, pode-se ver que acessibilidade financeira (50\%) e indicação por amigos ou parentes $(30 \%)$, justificam $80 \%$ das razões. Outras razões apresentadas foram: falta de assistência odontológica $(5 \%)$, insatisfação com outros serviços de atenção odontológica $(5 \%)$, razões ligadas a curiosidade (4\%), facilidade para ser atendido (3\%) e acessibilidade física da Faculdade $(1 \%)$, entre outras.

\section{3 - Opinião da clientela sobre 0 tratamento recebido na Facul- dade em 1973.}

A grande maioria entre os pacientes entrevistados (83\%; $n=263$ ), receberam atendimento aos problemas que os fizeram procurar a Faculdade. Porém, 16\% (n $=52$ ) somente passaram pelo Serviço de Triagem, não tendo recebido qualquer tipo de tratamento.

Considerando apenas os pacientes que foram atendidos (receberam algum tipo de tratamento), observouse que:

- $81 \%$ consultou mais de uma vez no ano de 1973 e, 19\% recebeu apenas uma consulta.

- três quartos (73\%) tiveram seus problemas dentários resolvidos, mas a solução não dependeu do número de consultas, provavelmente porque os tratamentos foram diferentes.

- as razões que predominaram, da não solução do problema na Faculdade, apresentadas pelos 72 pacientes, quase um terço dos que foram além da triagem (23\%), foram: situaçōes inerentes à Faculdade (51\%), seguidas de problemas pessoais $(25 \%)$ e abandono de tratamento (15\%). Considerando "problemas pessoais" também como "abandono de tratamento" lou vice-versa, "abandono" como "problemas pessoais"), verifica-se que 
isto constitui uma razão forte pois soma $40 \%$ dos casos não resolvidos. Assim, problemas inerentes à Faculdade ou ao paciente, justificam $92 \%$ dos casos de não solução do problema dentário que motivou a procura da Faculdade $121 \%$ do total dos entrevistados). Estes dois motivcs não são significantemente diferentes.

- a grande maioria (92\%) ficou satisfeita com o tratamento recebido (equivalendo a $76 \%$ dos que não retornaram). A satisfação está diretamente associada à solução do problema, pois $70 \%$ dos clientes estavam satisfeitos e com problema resolvido $(58 \%$ dos que não retornaram). Isto é uma situação desejável, embora pudesse ser melhor ainda. Assim, a ausência de total satisfação ou a não solução do problema dentário justificarão muito pouco a não reconsulta destes pacientes, durante 0 ano de 1974. É fato que, se fossem incluidos os que ficaram somente na triagem, a proporção de satisfatoriedade e solução do problema diminuiria para $58 \%$.

- para dois terços $165 \%$ ou 171 casos), não houve diferença entre o tratamento esperado e o recebido, enquanto que para um quarto houve diferença para melhor (67 casos). Estes dois grupos, em conjunto perfazem a grande maioria $180 \%$ entre os que foram além da triagem e $76 \%$ sobre o total de entrevistados).

Considerando-se a opinião de todos os pacientes que não retorna- ram $(N=315)$ sobre se completaram ou não o tratamento desejado na Faculdade, mais da metade $(56 \%)$ não completaram este tratamento. Entretanto, considerando apenas os pacientes que foram além da triagem ( $n=263$ ), pode-se admitir como igual o número de pacientes que completaram ou não o tratamento.

A solução do problema que motivou a procura da Faculdade está diretamente associada a conclusão do tratamento.

Assim, pelo menos alguns casos de não cura podem estar justificados pelos próprios pacientes em virtude de não concluírem o tratamento.

\section{4 - Razões para não reconsultarem na Faculdade de Odontologia em 1974. \\ - Proporção dos que não retorna- ram: \\ O número de pacientes registrados} em 1973 foi de 2.669 e destes, 2.392 não retornaram, conforme 0 arquivo da Faculdade. Entretanto, através da amostra estimou-se que $6,35 \%$ destes haviam retornado, sem entretanto, terem passado pelo Serviço de Triagem. Isto leva a uma estimativa de que 2.249 pacientes, dos registrados em 1973, não haviam retornado até fim de 1974, dando uma proporção de não-retorno, para o ano base da pesquisa, e nas condiçōes menciona. das, de $84,26 \%$. Isto é, cinco sextos da totalidade dos pacientes não reconsultaram.

Considerando que não há falta de clientes na Faculdade, estes dados confirmam a impressão de grande rotatividade de pacientes na mesma. Fatos desta natureza impedem a avaliação objetiva da eficácia dos traba. Ihos realizados, mesmo a curto prazo, 
pois apenas $16 \%$ dos pacientes retornam e somente sobre alguns destes é possível avaliação desta natureza.

- Razões para não reconsultarem em 1974

A tabela 6 apresenta a distribuição dos pacientes que não reconsultaram em 1974, conforme razões apresentadas.

Esta tabela encerra os atributos cuja grandeza comandaram o dimensionamento da aḿostra: as razões que, em conjunto, perfizessem a maior parte do não retorno em 1974, entre os pacientes que reconsultaram em 1973.

Pode-se observar que o não-retorno por: "problemas de horários", (falta de tempo do paciente ou incompatibilidade de horário), "não tinham problemas dentários" (na opinião dos pacientes), "procuraram outro dentista", perfazem 62,24\%, praticamente dois terços das razões apresentadas para o não retorno.

- Recebimento ou não de orientação para continuar o tratamento.

Observou-se que é significantemente maior o número de pacientes que afirmaram não terem recebido orientação $(53 \%$ equivalendo a $n=167$ ) sobre os que receberam $(38 \%, n=121)$ quando se considerou todos os pacientes que não retorna$\operatorname{ram}(\mathrm{N}=315)$.

\begin{tabular}{|c|c|c|c|}
\hline MOTIVOS & NÚMERO & $\begin{array}{l}\text { PROPORÇAO } \\
\%\end{array}$ & $\begin{array}{c}\text { PROPORCCÃO } \\
\text { ACUMULADA } \\
\% \\
\end{array}$ \\
\hline $\begin{array}{l}\text { Problemes de falta de tempo do } \\
\text { peciente; problemas de hordrio . . . }\end{array}$ & 90 & 28,58 & 28,58 \\
\hline N8̌o tinham problemss dentorios . . & 54 & 17,15 & 45,73 \\
\hline Procuraram outro dentista. . . . . & 52 & 16,51 & 62,24 \\
\hline $\begin{array}{l}\text { Perda de tempo na Faculdade } \\
\text { (demora para ser atendido; de- } \\
\text { mora do atendimento e/ou do } \\
\text { tratamento) } \ldots \ldots \ldots \ldots \ldots \ldots \ldots\end{array}$ & 26 & 8,25 & 70,49 \\
\hline $\begin{array}{l}\text { Problemes pessoais; doenca; aci- } \\
\text { dentes } \ldots \ldots \ldots \ldots \ldots \ldots \ldots \ldots\end{array}$ & 19 & 6,03 & 76,52 \\
\hline Conclurram o tratamento $\ldots \ldots$ & 17 & 5,40 & 81,92 \\
\hline $\begin{array}{l}\text { Insatisfacio com o tratamento } \\
\text { recebido } \ldots \ldots \ldots \ldots \ldots \ldots\end{array}$ & 14 & 4,44 & 86,36 \\
\hline $\begin{array}{l}\text { Negligencia; descuido; falta de } \\
\text { interesece } \ldots \ldots \ldots \ldots \ldots \ldots\end{array}$ & 14 & 4,44 & 90,80 \\
\hline Razóes económicas . . . . . . . . . & 6 & 1,90 & 92,70 \\
\hline Dificil acesso . . . . . . . . & 4 & 1,27 & 93,97 \\
\hline Falte de vago. . . . . . . . . . & 4 & 1,27 & 95,24 \\
\hline $\begin{array}{l}\text { Deseito de não trocar de protis. } \\
\text { sional. } \ldots \ldots \ldots \ldots \ldots \ldots \ldots\end{array}$ & 4 & $1,27^{\prime}$ & 96,51 \\
\hline Outros $\ldots \ldots \ldots \ldots \ldots$ & 11 & 3,49 & 100,00 \\
\hline TOTAL. . . . . . . . . . . & 315 & 100,00 & \\
\hline
\end{tabular}

R. Fac. Odont. P.A. 
- Busca de atendimento por outro dentista após atendimento na Faculdade em 1973.

Praticamente dois terços dos pacientes entrevistados procuraram outro dentista $(69 \%, n=218)$ que não os da Faculdade.

Como em outra parte deste trabaIho já se inferiu que "insatisfação" ou "não solução do problema" justificariam pouco do não-retorno, e agora se observa que a maioria procurou outro dentista, pode-se por coerência, começar a admitir que muitos destes pacientes assim tenham agido por disporem de outros serviços dos quais talvez não dispusessem no ano base da pesquisa.

Entre os que procuraram outro dentista pode-se ver que quase um terço consultou no próprio ano base da pesquisa (1973). Com relação ao tipo de dentista procurado, no total, tem-se que $96 \%$ procuraram dentista particular ou de serviço, sendo que: $70 \%$ procuraram dentista particular, $22 \%$ procuraram dentista de serviço e $4 \%$ procuraram a ambos (particular e de serviço).

Apesar de se ter admitido que a disponibilidade de outro dentista fosse importante para a procura de outro profissional, a maioria informou que procurou dentista particular (quase metade dos que não retornaram).

A localização da Faculdade não influiu na decisão de não retornar, na grande maioria dos casos $190 \%$ do total da amostra).

\section{5 - Posicionamento quanto a futu- ra reconsulta na Faculdade.}

Entre todos os pacientes, praticamente metade da amostra $151 \%$, $n=161)$ se posicionou favoravelmente a uma consulta futura na Faculda- de, enquanto um terço dos mesmos se posicionou desfavoravelmente $(34 \%, n=108) ; 14 \%$ ou deram respostas imprecisas ou não responderam.

As principais razões apontadas pelos favoráveis ao retorno foram: "se necessitarem de tratamento odontológico" (49\%) ou "porque apreciaram os tratamentos recebidos" (36\%), perfazendo $85 \%$ dos favoráveis a nova consulta $(43 \%$ do total de entrevistados).

Entre os 108 pacientes que são desfavoráveis a uma nova consulta futura na Faculdade, as razões que justificam a maior parte dos casos são: "possibilidade de procurar outro dentista (45\% entre os 108 e $16 \%$ sobre o total) "ausência de problemas dentários sentidos" (19\% entre os 108 e $6 \%$ sobre o total), "insatisfação com o tratamento recebido na Faculdade" (14\% entre os 108 e $5 \%$ do total). Estas três causas, no total, perfazem $79 \%$ entre os desfavoráveis e $27 \%$ entre os 315 que não retornaram.

Observou-se que há uma associação direta entre a solução do problema dentário que motivou a consulta e a tendência favorável de retornar a Faculdade.

\section{CONCLUSÕES}

Nas condições em que foi desenvolvido o estudo pode-se concluir com relação a população que se registrou no Serviço de Triagem da Faculdade de Odontologia na UFRGS em 1973 e não consultou em 1974, que:

- fato determinante associado à procura do Serviço Odontológico da Faculdade foi a acessibilidade financeira; 
- há rotatividade muito alta de pacientes no Serviço da Faculdade, com prejuízo de qualquer possibilidade objetiva de avaliação da eficácia dos trabaIhos realizados pelos estudantes;

- fato importante associado ao não-retorno foi a procura de outro dentista, geralmente particular, por não solução do problema na Faculdade;

- ao menos atualmente, a maioria tem disponibilidade de outros serviços odontológicos, predominantemente o INPS;

- entre os que não retornaram as principais causas que justificam esta conduta foram: falta de tempo, ausência de problemas dentários (subjetiva, na maioria das vezes), e fato de terem consultado outro dentista;

- a falta de recebimento de orientação para continuar o tratamento foi dominante e pode ter pesado na grande proporção de não-retorno;

- para ampliar a proporção de retorno possivelmente pesem favoravelmente atitudes como: - orientar os pacientes para o retorno a curto prazo, para continuar o tratamento ou para avaliação da situação clínica e determinação da conduta a seguir;

- manter a acessibilidade financeira;

- assegurar contínua supervisão para aumentar a eficácia do tratamento, buscando melhorar a eficácia do ponto subjetivo do paciente $\mathrm{e}$ objetivo do profissional;
- talvez maior ênfase na parte social de recebimento e orientação do paciente, esta tanto durante a triagem quanto ao término de cada atendimento;

- redução no tempo de atendimento, em termos de espera na Faculdade e de intervalo entre as consultas;

- exame geral oral, indicando aos pacientes outros problemas existentes, além dos sentidos por eles.

\section{SUMARY}

Three hundred fifteen patients who searched dental treatment at UFRGS dental clinics in 1973 and who did not return in 1974 were interviewed and the main reasons given were recorded. Relevant factors were analysed, such as: personal data, socio-economic status, factors that have influenced the utilization of clinical services at the University, and attitudes regarding the treatment received and future treatment at the University. Various associations of these factors and their combinations were also analyzed. The main reasons given by the patients for not returning were: they didn't have time, they needed no treatment and they went to another dentist. 


\section{REFERÊNCIAS BIBLIOGRÁFICAS}

1. ABRAMOWICZ, Mendel; GIL Carlos; MARTINS, Maria do Carmo Bottino. Contribuição para o estudo dos pacientes que freqüentam as clínicas da Faculdade de Odontologia da Universidade de São Paulo. Revista da Faculdade de Opontologia da Universidade de São Paulo, São Paulo, 14(2) :259-70, Jul./Dez. 1976.

2. BRYNOLF, Ingrid. The socio-economic background of the patients at the Royal Dental School, Malmö. Odontologisk Rev, Malmö, 17(3):27485, 1966.

3. DOUGLAS, Bruce L. \& COHEN, Lawrence. The teaching patients population in dental education. Inquiry, Chicago, 10:33-41, Mar. 1973.

4. DWORKIN, S.F. et alii. Factors contributing to patient attrition at a University Dental Center. I.A.D.R. Abstracts; Program and Abstracts of Papers, New York, 48:188 (Abstract 558), 1970.

5. GREWE, John M.; GORLIN, Robert J.; MESKIN, Lawrence H. The dental patient at the University of Minnesota: characterization. Journal of Dental Education, Houston, 32(4):420-9, 1968.

6. KOSLOWSKY, Meni; BAILIT, Howard; VALLUZZO, Paula. Satisfaction of the patient and the provider; evaluation by questionnaire. Journal of Public Health Dentistry, Raleigh, 34(3):188-94, 1974.

7. KRESS Jr., Gerard C.; FERRARO, Eugenen; STIFF, Robert. Patients' evaluation of outpatient services in a school of dental medicine. Journal of Public Health Dentistry, Raleigh, 33(2): 104-9, 1973.

8. MESKIN, Lawrence $H$. Current and future problems in obtaining teaching patients. Journal of Dental Education, Chicago, 33(1):112-8, 1969.

9. PONTIFÍCIA UNIVERSIDADE CATÓLICA DO RIO GRANDE DO SUL. Instituto de Estudos Sociais, Políticos e Econômicos. Coordenação de Pesquisa Aplicada. O sistema educacional e o mercado de trabalho na regiáo metropolitana de Porto Alegre (Projeto 01); relatório de pesquisa. Porto Alegre, 1976, 226p.

10. SOULE, Doug J.; HORTON, PhillipS.; STEINHAUSER, Anthony D. Why patients select dental schools for treatment: effects of fees structure and student appearance. Journal of Dental Education, Chicago, 38(11):638-41, Nov. 1974. 